\title{
Serum soluble transferrin receptor concentration is an accurate estimate of the mass of tissue receptors
}

\author{
Samir R'zik and Yves Beguin \\ Department of Medicine, Division of Hematology, University of Liège, Liège, Belgium
}

(Received 18 October 2000; revised 29 December 2000; accepted 24 January 2001)

\begin{abstract}
Objective. Serum levels of the soluble transferrin receptor (sTfR) vary depending on the erythropoietic activity and iron status. In vitro, sTfR shed in the incubation medium correlates well with cellular TfR, but this relationship has never been established in vivo. To determine the value of serum sTfR as a quantitative marker of the body mass of tissue TfR, we designed experiments to examine the correlation between serum sTfR and tissue TfR in rats with various degrees of erythropoietic activity or iron status.

Materials and Methods. We studied changes in erythropoietic activity in normal rats as well as in animals experiencing hemolysis, phlebotomy-induced iron deficiency, transfusion- or thiamphenicol-induced erythroid aplasia, or inflammation. At the end of follow-up, ferrokinetic studies were performed and animals were sacrificed. Organs were isolated and homogenized to determine the total mass of tissue TfR from the sum of tissue solubilized TfR in the bone marrow, spleen, liver, and blood cells (direct method). An indirect method was developed to derive the corporeal mass of tissue TfR from a representative marrow sample.

Results. As expected, serum sTfR and total mass of tissue TfR varied as a function of iron status and erythropoiesis. Relative erythroid expansion in the spleen was greater than in the bone marrow. With the exception of phlebotomized animals, the indirect method correlated very well with direct measurements of the total mass of tissue $\operatorname{TfR}(r=0.97, p<0.0001)$. There was a close relationship between the total mass of tissue TfR and the total mass of serum sTfR $(r=$ $0.79, p<0.0001)$. Serum sTfR represented approximately 5-6\% of the total mass of tissue TfR in most experimental situations, but this ratio was twice as high during iron-restricted erythropoiesis. In addition, the ratio could be higher or lower in nonsteady-state situations, because changes in tissue TfR occurred faster than those of serum sTfR.

Conclusions. Serum sTfR represents a constant proportion of the total mass of tissue TfR over a wide range of erythropoietic activity. However, iron deficiency results in a higher proportion of serum sTfR, and the pace of change in serum sTfR levels is slower than that of tissue TfR mass. (c) 2001 International Society for Experimental Hematology. Published by Elsevier Science Inc.
\end{abstract}

Iron transport in the plasma is carried out by transferrin, which donates iron to cells through its interaction with a specific membrane receptor, the transferrin receptor (TfR) [1-3]. The functional receptor is composed of two monomers linked by two disulfide bridges to form a molecule of 190,000 Da. Virtually all cells, except mature red cells, have TfR on their surface, but the largest numbers are in the erythron, placenta, and liver. Many more TfR are seen on colony-forming unit-erythroid than on burst-forming unit-

Offprint requests to: Yves Beguin, M.D., Department of Hematology, University of Liège, CHU Sart-Tilman, 4000 Liège, Belgium; E-mail: yves.beguin@chu.ulg.ac.be erythroid [4], and their numbers increase to about 300,000 on early normoblasts and up to 800,000 on intermediate normoblasts [5], before declining to about 100,000 on reticulocytes and none on mature red cells [5,6]. A soluble form of TfR (sTfR) has been identified in animal [7] and human serum [8]. sTfR is a truncated monomer of tissue receptor, lacking its first 100 amino acids, which circulates in the form of a complex of transferrin and its receptor [7-9]. The most important determinant of serum sTfR levels appears to be marrow erythropoietic activity, which can cause variations up to $8 \times$ below and up to $20 \times$ above average normal values $[7,8]$. Serum sTfR levels are decreased in situations characterized by diminished erythropoietic activity and are increased when erythropoiesis is stimulated. Iron status also 
influences serum sTfR levels, which are considerably elevated in iron deficiency anemia and already so in functional iron deficiency, a situation defined by tissue iron deficiency despite adequate iron stores $[10,11]$.

Before the discovery of serum sTfR, erythron transferrin uptake (ETU), a refined ferrokinetic measurement of the number of transferrin molecules taken up by erythroid TfR per day, had been validated as the best available quantitative measurement of total erythropoietic activity, i.e., of the total mass of TfR-bearing erythroblasts $[12,13]$. We previously showed that there was a remarkable correlation between ETU and serum sTfR levels in rats [7] and in humans [8] over a wide range of erythropoietic activity. However, ETU is no longer valid when cellular TfR are not saturated by iron-bearing transferrin, this being observed when the transferrin saturation is less than $20 \%$. It is not known whether serum sTfR then would remain a valid measure of the cellular TfR mass. Although experiments with leukemic cell lines have demonstrated an excellent correlation between cellular TfR and sTfR in the incubation medium [14,15], this relationship has never been established in vivo for normal erythroid cells. Providing such would definitively ascertain the value of serum sTfR as a quantitative marker of the body mass of tissue TfR. Therefore, we designed simple experiments to examine the correlation between serum sTfR and the mass of tissue TfR in rats with various degrees of erythropoietic activity or iron status.

\section{Materials and methods}

\section{Animals}

Male Wistar rats were obtained from Janssen (Beerse, Belgium) and raised at the University of Liège from the age of 3 weeks through the end of the experiment. Animals were fed standard granular food without iron supplements. All experiments were carried out under ether anesthesia. Hemolytic anemia was produced by intraperitoneal injection of $50 \mathrm{mg}$ acetylphenylhydrazine per kilogram body weight. Some animals received a single dose on day 0 , and others received three doses on days 0,4 , and 8 to obtain on-going hemolysis. Red cell aplasia was provoked by intraperitoneal injections of thiamphenicol in one of three different protocols: $50 \mathrm{mg}$ b.i.d. (twice daily) for 4 consecutive days; $100 \mathrm{mg}$ once daily for 10 consecutive days; or $100 \mathrm{mg}$ on days $0,3,6,9$ and 12. Inflammation was generated by injecting $0.25 \mathrm{~mL}$ of turpentine oil in each thigh. Iron-deficient erythropoiesis was induced by bleeding animals $3-4 \mathrm{~mL}$ on 2 consecutive days and then on alternate days thereafter to maintain a hematocrit (Hct) of about 25\%-30\%. To suppress erythropoiesis, rats were transfused with 3-4 mL of washed packed red cells every other day to maintain a hematocrit of 60-70\%. Donor animals were previously treated with thiamphenicol to induce red cell aplasia and thus procure blood with less than $2 \%$ reticulocytes.

\section{Experimental procedures}

Blood samples were drawn from a tail vein $3 \times$ per week until the final day of the experiment, when ferrokinetic studies were performed. Labeling with ${ }^{59} \mathrm{Fe}$ was accomplished by adding $1 \mu \mathrm{Ci}$ ${ }^{59} \mathrm{Fe}$ citrate to $0.5 \mathrm{~mL}$ of donor plasma under continuous agitation.
The plasma was incubated for 30 minutes at $37^{\circ} \mathrm{C}$ before injection into a tail vein. Blood samples were obtained from the tail vein on the opposite side, and the rate of radioiron disappearance from circulation $\left(\mathrm{t}_{1 / 2}\right)$ was determined by least squares regression analysis. Based on the plasma iron (PI), Hct, and clearance half-time of ${ }^{59} \mathrm{Fe}$ $\left(\mathrm{t}_{1 / 2}\right)$, plasma iron turnover (PIT, $\mathrm{mg} / \mathrm{dL}$ whole blood/day) was calculated from a standard formula given in Equation 1 [16]:

$$
\begin{aligned}
& \text { PIT }(\mathrm{mg} / \mathrm{dL} \text { whole blood/day })= \\
& \frac{\mathrm{PI}(\mu \mathrm{g} / \mathrm{DL}) \times(100-\text { Hct } \times 0.9) / 100}{\mathrm{t}_{1 / 2}(\min )}
\end{aligned}
$$

where 0.9 is the correction for trapped plasma in the hematocrit (0.98) and to convert venous hematocrit to whole body hematocrit (0.92). The extravascular flux, a function of plasma iron concentration [16], was subtracted to obtain tissue iron uptake (TIU), as given in Equation 2: [PI $\times(100-\operatorname{Hct} \times 0.9) / 100 \times 0.0015]$.

$$
\begin{aligned}
& \text { TIU }(\mathrm{mg} / \mathrm{dl} \text { whole blood/day })=\mathrm{PIT}- \\
& {[\mathrm{PI} \times(100-\mathrm{Hct} \times 0.9) / 100 \times 0.0015] .}
\end{aligned}
$$

The effect of transferrin saturation $(\mathrm{S})$ was removed by converting TIU to iron-bearing transferrin uptake, and nonerythron uptake was subtracted [12] to obtain erythron transferrin uptake (ETU), as given in Equation 3 [12,13]:

$$
\begin{aligned}
& \text { ETU }(\mu \mathrm{M} / \mathrm{L} \text { whole blood/day })= \\
& \frac{\text { TIU } \times 10}{56} \times \frac{(200+2.2 \mathrm{~S})}{(200+6.4 \mathrm{~S})}-11 .
\end{aligned}
$$

Three hours (6 hours for hypertransfused animals) after radioiron injection, rats were exsanguinated from the abdominal aorta and perfused with $20--30 \mathrm{~mL}$ of saline. The spleen, liver, and two femurs were removed and weighted. Whole blood and plasma radioactivities, as well as that of liver, spleen, and femurs, were counted. Total plasma and total red cell radioactivities were calculated from the hematocrit, assuming a blood volume of $0.07 \mathrm{~mL} / \mathrm{g}$ body weight [17]. Erythroid marrow radioactivity was estimated by multiplying the counts of the two femurs by 6.5 , because marrow in one femur represents $1 / 13$ of total bone marrow in the rat [18]. The bone marrow was extracted by 1) flushing out of the femur shafts and 2) grinding the two femur extremities and recovering the supernatant after centrifugation. These two marrow components were combined and counted. The efficiency of marrow extraction was calculated as the ratio of radioactivity recovered to the radioactivity in the unmanipulated femurs (average $56 \% \pm 8 \%$ ). All calculations of marrow TfR were corrected for the efficiency of marrow extraction determined for each individual animal.

The whole spleen and extracted bone marrow, weighted aliquots of the liver, and washed cells from $1 \mathrm{~mL}$ of whole blood were homogenized and transferrin receptors solubilized at $4{ }^{\circ} \mathrm{C}$ in a Polytron PT-K homogenizer (Kinematica, Littau, Switzerland) for 1 minute at $15,000 \mathrm{rpm}$ and then $0.5-2$ minutes at 24,000 rpm. All samples were homogenized in adequate amounts of $10 \mathrm{mM}$ phosphate-buffered saline, $\mathrm{pH} 7.4$, containing $1 \mathrm{mM}$ iodoacetic acid, $0.5 \mathrm{mM}$ PMSF, $20 \mathrm{U} / \mathrm{mL}$ aprotinin, and $2 \%$ of the nonionic detergent TERIC (Sigma Chemical Corp., St. Louis, MO, USA). After homogenization, samples were centrifuged for 35 minutes at $51,000 \mathrm{~g}$ at $4^{\circ} \mathrm{C}$ in a Supraspeed RC28S Sorvall centrifuge (Sorvall Instruments, Du Pont Company, Wilmington, DE, USA). The supernatants were collected and counted to calculate the percentage recovery of the original sample (ratio of radioactivity in homoge- 
nate to that of corresponding original sample), which ranged from $59 \% \pm 18 \%$ for blood cells to $77 \% \pm 6 \%$ for bone marrow. The recovery of blood cell TfR was lower because of the radioactivity trapped in the large volume of precipitated membrane ghosts and hemoglobin. All calculations of solubilized tissue TfR were corrected for this percentage recovery determined for each individual sample. Solubilized tissue TfR concentration was measured on fresh supernatants of each homogenized organ.

\section{Laboratory analyses}

Blood samples were drawn from a tail vein. Part of the blood was drawn on citric acid, sodium citrate, dextrose (ACD) formula A and part on heparin. Heparinized blood was centrifuged to obtain plasma that was frozen at $-20^{\circ} \mathrm{C}$ until processing. Hematocrits were determined by the micromethod technique. Complete blood counts were measured on a Technicon $\mathrm{H} 1$ automatic cell counter (Tarrytown, NY, USA) with appropriate corrections for dilution by ACD. The percentage of reticulocytes was determined by cytofluorometry on a FACSCAN cytofluorometer (Becton-Dickinson, San Jose, CA, USA) after coloration with thiazole orange [19]. Preliminary experiments showed that this automated method gave results similar to manual counting on blood smears colored with brilliant cresyl blue. Serum sTfR and solubilized tissue TfR were measured by ELISA, as previously described with minor modifications $[7,20]$. Serum iron ( $\mathrm{SeFe}$ ) and total iron-binding capacity (TIBC) were measured by standard methods [21], and transferrin saturation was derived from these figures.

\section{Calculation of the mass of TfR}

The concentration of solubilized tissue TfR measured in the supernatant of each organ homogenate was corrected for recovery of radioactivity counted in the original sample and expressed per milliliter (blood cells and marrow) or gram (liver and spleen) of organ (see earlier). The total mass of TfR in each organ was calculated based on this concentration and the total weight (liver and spleen) or volume (plasma, blood cells, and marrow) of the organ considered, taking into account the efficiency of organ extraction for the bone marrow (see earlier) and multiplying the mass of the two femurs by 6.5 to obtain the total TfR mass in the bone marrow. The total body mass of tissue TfR ("total TfR mass") was calculated as the sum of the total masses of TfR in liver, spleen, marrow, and blood cells.

The total mass of tissue TfR also was measured by an indirect method in which this total mass is derived from a simple representative sample ("corporeal TfR mass"). A bone marrow sample (i.e., femur bone marrow) is an adequate representative of all tissue TfR if injected radioiron has similar access to TfR of all organs. Radioactivity of such a sample truly represents radioiron uptake by marrow TfR if there is no contamination by blood radioactivity. To ensure this, the timing of evaluation must be such that serum radioactivity has virtually disappeared and red cell radioactivity from newly synthesized erythroblasts has not yet left the bone marrow. Thus, after injection of a known total amount of radioactivity, measurements of radioactivity and solubilized TfR in the representative sample supernatant allow calculation of the corporeal TfR mass, based on the formula given in Equation 4:

Corporeal TfR mass $(\mu \mathrm{g})=$

Solubilized tissue TfR $(\mathrm{ng} / \mathrm{mL})$ in sample $\times$

$\frac{\text { Total radioactivity injected }(\mathrm{cpm})}{\text { Radioactivity of sample }(\mathrm{cpm} / \mathrm{mL})} \times 10^{-3}$.
Statistical methods

All results are presented as mean \pm standard deviation $(\mathrm{M} \pm \mathrm{SD})$ and expressed either in absolute values or as percentages of a baseline value. Comparisons of baseline values with later measurements in the same group of animals were carried out by paired Student's $t$-tests. Comparisons between groups were carried out with Student's $t$-tests, with Welsch's correction in case of unequal variances. The $r$ correlation coefficients between two variables were computed using least squares regression equations. Most statistical analyses were carried out using Excel 97 (Microsoft Corporation, Redmond, WA, USA) or Prism 2.0 (GraphPad Software Inc., San Diego, CA, USA) softwares.

\section{Results}

Normal animals followed-up for 16 days with blood samples drawn on alternate days showed no significant changes in Hct, reticulocytes, or serum STfR throughout the experiment (data not shown). Compared to rats sacrificed on day 0 , animals studied after a 16-day experiment had similar values for $\mathrm{SeFe}$, TIBC, transferrin saturation, ${ }^{59} \mathrm{Fe} \mathrm{t}_{1 / 2}, \mathrm{PIT}$, and ETU, as well as total mass of tissue TfR in the various organs and in the total body. This demonstrates that the experiment by itself did not induce significant changes in erythropoietic activity or iron metabolism.

After an acute hemolysis caused by phenylhydrazine, the response of the erythroid compartment to the anemia became rapidly apparent with peak reticulocytosis on day 5 and peak serum sTfR values on day 8 (Fig. 1A). The absolute increase in the mass of tissue TfR was similar in the spleen and bone marrow (Table 1). However, the relative expansion of splenic TfR mass (almost 12-fold baseline) was greater than that of the bone marrow TfR mass (2.5fold baseline) (Table 1). This resulted in correction of the anemia by day 8 (Fig. 1A). As iron remained readily available, the ETU increased in proportion to the augmented TfR mass of the erythron (Table 1). In rats receiving three injections of phenylhydrazine on days 0,4 , and 8, elevated reticulocytes and serum sTfR levels were maintained through the end of the experiment on day 14 (data not shown), resulting in compensated hemolysis, i.e., correction of anemia by day 12 despite continuing red blood cell destruction. The expansion (19-fold) of splenic but not that of marrow TfR mass was even more prominent than after a single injection.

The anemia maintained by repeated phlebotomies was followed by a rapid erythroid response, as illustrated by considerably elevated reticulocytes and serum sTfR (Fig. 1B). The bone marrow could only double its TfR mass, whereas the expansion reached 12-fold in the spleen (Table 1). However, erythropoiesis rapidly became iron restricted, with extremely low SeFe and transferrin saturation and increased TIBC. Major increases in tissue TfR were observed not only in the erythron but also in the liver, reflecting the development of tissue iron deficiency. As a result, PIT and ETU became gross underestimates of the mass of tissue TfR.

Hypertransfusion resulted in polycythemia-induced red 



B

Hct
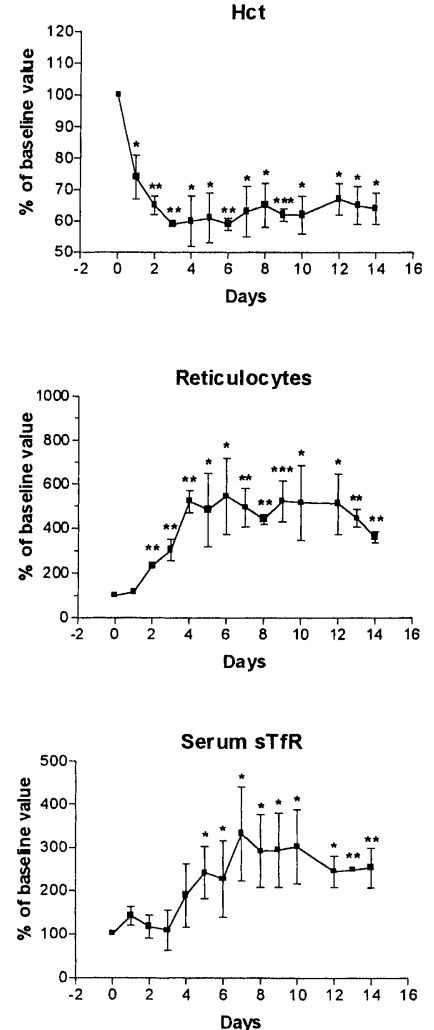
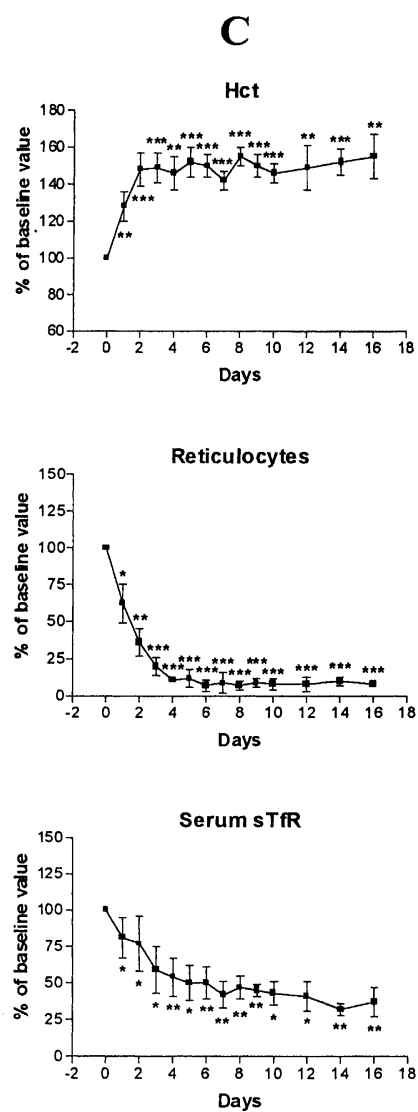

Figure 1. Evolution of hematocrit (Hct), reticulocytes, and serum sTfR levels in groups of four rats experiencing hemolysis induced by acetylphenylhydrazine on day 0 (A), repeatedly phlebotomized to maintain Hct around 25-30\% (B), and hypertransfused with reticulocyte-poor blood to maintain Hct around $60-70 \%(\mathbf{C})$.

cell aplasia (Fig. 1C). Maximal suppression of the erythroid compartment was already achieved by day 5 (Table 2), but serum sTfR continued to decrease slowly to stabilize only later. ETU, but not PIT, accurately reflected the reduction in total mass of tissue TfR. The contribution of nonerythroid tissues, the liver, as well as other organs not analyzed here, to the diminished total mass of tissue and thus serum sTfR became more prominent.

Animals treated with thiamphenicol twice daily for 4 days experienced a substantial degree of erythroid suppression, then active regeneration with reticulocytes and serum sTfR overshooting baseline levels to produce supranormal Hct values (Fig. 2A). The mass of tissue TfR was profoundly constrained on day 4 but rebounded to more than $3 \times$ normal levels by day 7 and remained above normal through day 18 (Table 2). Limited iron mobilization, illustrated by low Tf saturation and normal PIT and ETU despite increased tissue TfR numbers, probably restricted the expansion of erythropoiesis. Daily injections of thiamphenicol for 10 days (data not shown) produced a prolonged suppression of erythropoiesis (low reticulocytes, serum sTfR, tissue TfR, and ETU) and thus a more substantial degree of ane- mia (70\% of baseline). The pattern of regeneration was similar but amplified compared to the shorter experiment. On the other hand, intermittent treatment on days $0,3,6,9$, and 12 did not bring about anemia.

Acute inflammation caused modest decrements of reticulocyte and serum sTfR values resulting in a moderate degree of anemia after 48 hours, followed by a progressive correction thereafter (Fig. 2B). Tf saturation and the PIT were decreased on day 1 , but no other significant change in tissue TfR or ferrokinetic data was observed on days 1,2 , or 4 (data not shown).

For each organ, the blood cells $(r=0.60, p<0.001)$, bone marrow $(r=0.58, p<0.001)$, spleen $(r=0.73, p<$ $0.001)$ and liver $(r=0.73, p<0.001)$, there was a strong correlation between the fraction of injected radioactivity taken up and the relative contribution of that organ to the total mass of tissue TfR. In normal animals, the fraction of injected radioiron taken up in each organ was almost identical to the proportion of body tissue TfR in that organ. However, in situations of intense stimulation of erythropoiesis, radioiron uptake by blood cells became more in excess of their relative contribution to the total tissue TfR mass. For in- 


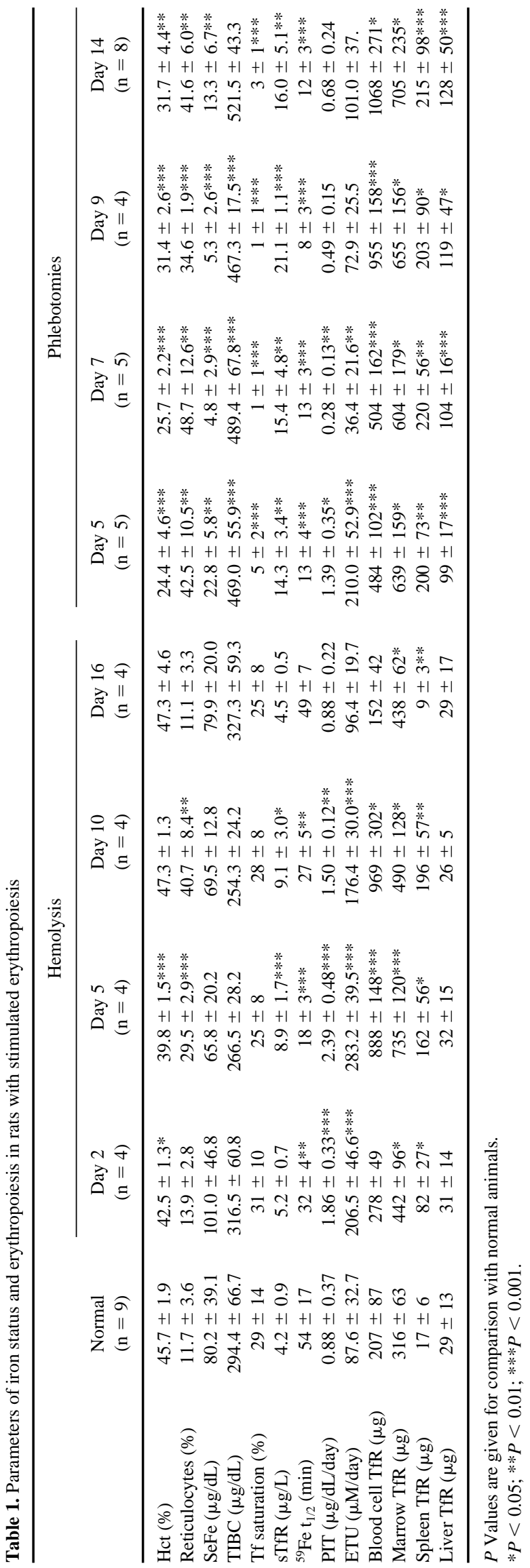

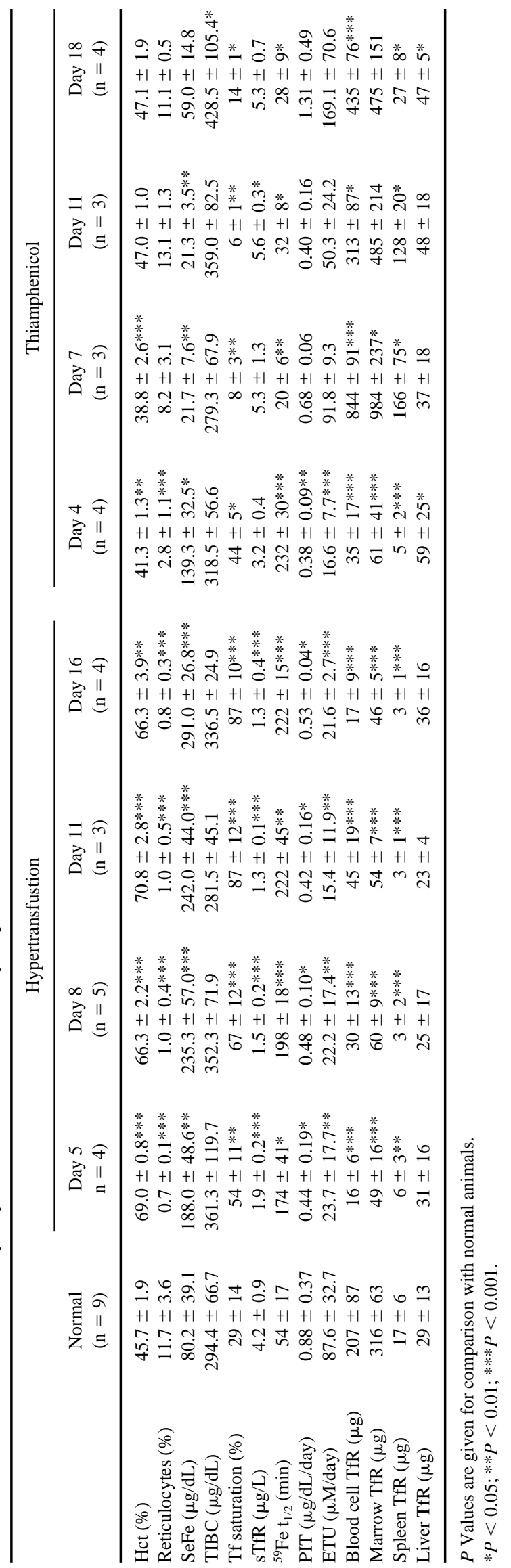



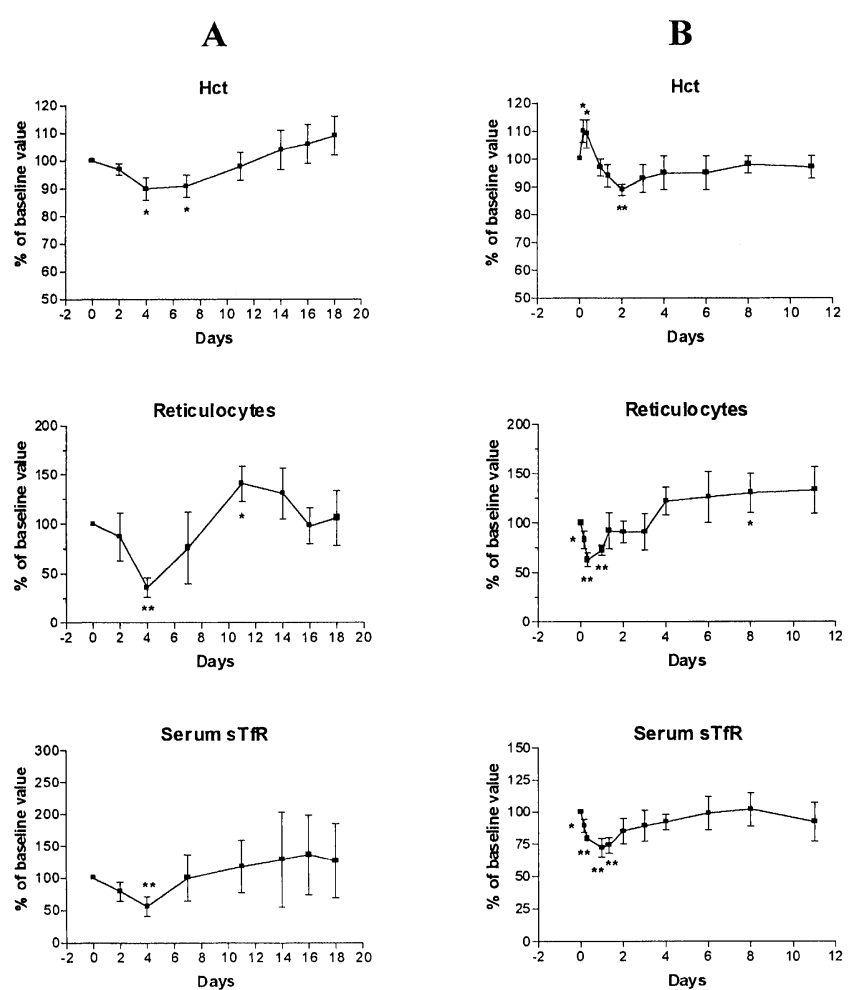

Figure 2. Evolution of hematocrit (Hct), reticulocytes, and serum sTfR levels in groups of four rats treated with thiamphenicol for 4 days (A) and experiencing acute inflammation induced by turpentine oil on day 0 (B).

stance, in normal rats $31 \% \pm 7 \%$ of the radioactivity went to blood cells $(35 \% \pm 8 \%$ of total tissue TfR mass) and $53 \% \pm 3 \%$ to bone marrow $(57 \% \pm 8 \%$ of total tissue TfR mass), whereas in day 9 phlebotomized animals $68 \% \pm 3 \%$ of radioactivity went to blood cells $(49 \% \pm 8 \%$ of total tissue TfR mass) and $16 \% \pm 8 \%$ to bone marrow $(34 \% \pm 8 \%$ of total tissue TfR mass).

The corporeal TfR mass represents an indirect method for estimating the total mass of tissue TfR from the total dose of radioactivity injected and measurements of radioactivity and solubilized tissue TfR in a representative marrow sample (see Materials and methods). In phlebotomized animals, the indirect method yielded vastly exaggerated values for total body tissue TfR, 2- to 2.5-fold higher than the value obtained after direct measurements in the various organs. In all other categories of animals, the indirect method gave excellent results that correlated extremely well ( $r=$ 0.97, $p<0001$ ) with those of direct measurements (Fig. 3).

The total mass of serum TfR was calculated from the serum STfR concentration and plasma volume and ranged from $8 \pm 1 \mu \mathrm{g}$ in hypertransfused animals to $199 \pm 18 \mu \mathrm{g}$ in phlebotomized rats after 9 days (Table 3 ). The correlation between ETU and the total mass of serum sTfR was excellent ( $r=0.84, p<0.001)$, but only when animals with very low (phlebotomy, recovery after thiamphenicol treatment)

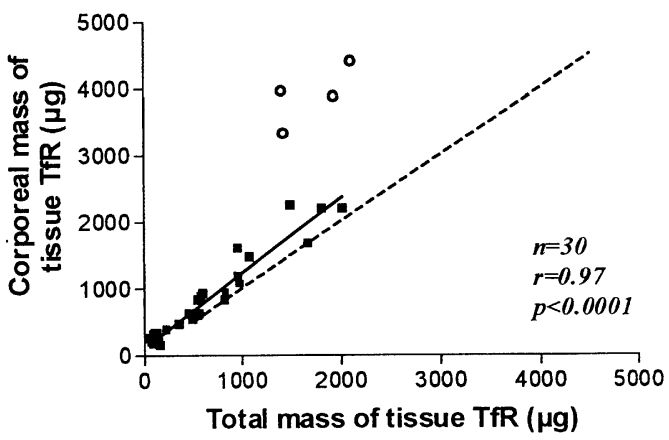

Figure 3. Correlation between the total mass of tissue TfR (obtained by direct measurement in the various organs) and the corporeal mass of tissue TfR (calculated from the analysis of a representative marrow sample after radioiron injection). Each closed square represent a group of 3-6 animals studied on a given day in a given condition. Open circles denote groups of phlebotomized animals excluded from the correlation because the indirect method was not adequate for them (see text).

or very high (hypertransfusion) SeFe levels were excluded. The correlation between ETU and the total body mass of tissue TfR was as good $(r=0.90, p<0.001)$, provided the same restrictions were applied (Fig. 4A). Hence, we could establish a close relationship between the total mass of tissue TfR and the total mass of serum sTfR $(r=0.79, p<$ 0.0001 ) (Fig. 4B). The correlation with the total mass of tissue TfR was maintained when the serum sTfR concentration was used instead of the total mass of serum sTfR ( $r=$ $0.78, p<0.0001)$. The total mass of serum sTfR represented approximately 5-6\% of the total mass of tissue TfR in normal animals, and this proportion was preserved in most experimental situations (Table 3). However, this ratio was consistently higher (around 10\%) during iron-restricted erythropoiesis induced by phlebotomies. In addition, the ratio was much higher on day $4(16 \%)$ but much lower on day $7(2 \%)$ of thiamphenicol treatment. Finally, after hypertransfusion, the ratio initially increased and only progressively returned to normal values because changes in tissue TfR occurred faster than those of serum sTfR.

\section{Discussion}

In normal animals, $95 \%$ of body tissue TfR was localized in the erythron, but this is probably an overestimate because the modest contribution of nonerythroid organs other than the liver was not evaluated. As mature red cells bear no TfR and white blood cells are much less numerous and express much fewer TfR, the blood cell TfR mass is indeed the reticulocyte TfR mass. Tissue TfR numbers reflect cellular iron demand and increase when either the number of TfRbearing cells (mostly reticulocytes and erythroblasts in erythroid tissues) increases or the number of TfR per cell increases (as in iron deficiency). Thus, in the absence of iron deficiency, the mass of tissue TfR represents the rate of 
Table 3. Relationship of the total mass of serum sTfR to the total mass of tissue TfR in rats under various conditions

\begin{tabular}{|c|c|c|c|c|c|}
\hline & & $\mathrm{N}$ & Total mass of serum sTfR $(\mu \mathrm{g})$ & Total mass of tissue TfR $(\mu \mathrm{g})$ & Serum sTfR/tissue TfR (\%) \\
\hline Normal & Day 0 & 9 & $37.0 \pm 9.6$ & $568.7 \pm 143.6$ & $6.7 \pm 1.8$ \\
\hline \multirow[t]{4}{*}{ Hemolysis $(\mathrm{PNH} \times 1)$} & Day 2 & 4 & $47.1 \pm 6.6$ & $833.8 \pm 142.8^{*}$ & $5.7 \pm 0.8$ \\
\hline & Day 5 & 4 & $83.3 \pm 14.8^{* * *}$ & $1816.2 \pm 144.6^{* * *}$ & $4.6 \pm 0.9$ \\
\hline & Day 10 & 4 & $77.1 \pm 28.2 *$ & $1681.8 \pm 459.4^{*}$ & $4.6 \pm 0.9$ \\
\hline & Day 16 & 4 & $42.7 \pm 1.5$ & $627.8 \pm 108.7$ & $7.0 \pm 1.2$ \\
\hline \multirow[t]{2}{*}{ Hemolysis $(\mathrm{PNH} \times 3)$} & Day 14 & 3 & $82.0 \pm 12.7 * * *$ & $1496.9 \pm 116.8^{* * *}$ & $5.5 \pm 0.4$ \\
\hline & Day 18 & 3 & $67.2 \pm 6.8 * *$ & $957.1 \pm 46.3^{* *}$ & $7.0 \pm 0.4$ \\
\hline \multirow[t]{4}{*}{ Phlebotomy } & Day 5 & 5 & $161.3 \pm 42.6^{* *}$ & $1421.3 \pm 180.4^{* * *}$ & $11.8 \pm 4.4^{*}$ \\
\hline & Day 7 & 5 & $163.9 \pm 51.8^{* *}$ & $1430.6 \pm 344.1 * *$ & $11.3 \pm 1.1 * * *$ \\
\hline & Day 9 & 4 & $199.1 \pm 17.5^{* * *}$ & $1932.6 \pm 353.2 * *$ & $10.6 \pm 2.0^{* *}$ \\
\hline & Day 14 & 8 & $195.1 \pm 39.7 * * *$ & $2116.2 \pm 387.8^{* * *}$ & $10.0 \pm 2.2 * *$ \\
\hline \multirow[t]{4}{*}{ Hypertransfusion } & Day 5 & 4 & $11.1 \pm 1.2 * * *$ & $101.9 \pm 11.7 * * *$ & $10.9 \pm 0.8^{* *}$ \\
\hline & Day 8 & 4 & $10.6 \pm 2.0^{* * *}$ & $116.9 \pm 21.5^{* * *}$ & $9.2 \pm 1.5^{*}$ \\
\hline & Day 11 & 4 & $8.1 \pm 0.8^{* * *}$ & $125.6 \pm 25.5^{* * *}$ & $6.6 \pm 0.7$ \\
\hline & Day 16 & 4 & $8.3 \pm 2.2 * * *$ & $102.4 \pm 21.3^{* * *}$ & $7.9 \pm 2.5$ \\
\hline \multirow[t]{4}{*}{ Thiamphenicol (4 days) } & Day 4 & 4 & $28.7 \pm 4.2$ & $160.2 \pm 63.1^{* * *}$ & $15.5 \pm 3.1^{* * *}$ \\
\hline & Day 7 & 3 & $47.0 \pm 9.0$ & $2030.5 \pm 128.7 * * *$ & $2.3 \pm 0.6 * *$ \\
\hline & Day 11 & 3 & $48.1 \pm 1.6^{*}$ & $974.8 \pm 287.5^{* * *}$ & $5.2 \pm 1.5$ \\
\hline & Day 18 & 4 & $53.1 \pm 10.3^{*}$ & $984.3 \pm 140.7 * * *$ & $5.4 \pm 0.9$ \\
\hline Thiamphenicol (9 days) & Day 10 & 3 & $12.6 \pm 3.8^{*}$ & $152.5 \pm 39.6^{* *}$ & $8.2 \pm 0.5$ \\
\hline \multirow[t]{3}{*}{ Inflammation } & Day 1 & 4 & $29.7 \pm 2.3$ & $545.3 \pm 86.7$ & $5.5 \pm 0.8$ \\
\hline & Day 2 & 4 & $45.4 \pm 6.8$ & $543.4 \pm 216.5$ & $9.0 \pm 2.0$ \\
\hline & Day 4 & 4 & $42.2 \pm 7.3$ & $576.3 \pm 191.8$ & $8.3 \pm 3.9$ \\
\hline
\end{tabular}

$\mathrm{PNH}=$ phenylhydrazine.

$* P<0.05 ; * * P<0.01 ; * * * P<0.001$.

erythropoietic activity. The total mass of tissue TfR in the erythron showed considerable plasticity, covering a 28 -fold variation from polycythemia-induced red cell aplasia to iron-deficient anemia caused by repeated phlebotomies. The spleen contributed considerably to the expansion of erythropoiesis, up to $50 \%$ of the total expansion of the tissue TfR mass and up to 19-fold over its own baseline activity, whereas the bone marrow attained its physiologic limit much sooner $[22,23]$. This already was reported in situations of hypoxia or hemolysis- or phlebotomy-induced anemia [24-26]. The mass of liver TfR remained constant in most experimental conditions studied here, except after phlebotomies. The latter is characterized by tissue iron deficiency and thus increased expression of TfR in all cell types $[2,27]$. On the other hand, iron-restricted erythropoiesis or functional iron deficiency as produced by erythroid regeneration after thiamphenicol-induced red cell aplasia may cause a more modest increase in liver TfR expression despite presumably maintained iron stores. Finally, iron overload brought about by repeated transfusions did not translate into a significant reduction in liver TfR expression. The moderate elevation of the liver TfR mass on day 4 of thiamphenicol treatment remains unexplained but could be an effect of the drug itself.

The indirect method of estimating the total mass of tissue TfR based on a representative marrow sample gave excellent results that correlated extremely well with those of the direct measurements. This implies that the contribution of organs not examined here was minimal. This validation of the indirect methodology makes it applicable to humans, in whom direct measurements are impossible for obvious ethical reasons. However, the indirect method yielded vastly exaggerated values for total body tissue TfR in phlebotomized animals, 2- to 2.5 -fold higher than the value obtained after direct measurements in the various organs. These rats were characterized by massive reticulocytosis and severely iron-restricted erythropoiesis. In addition, in situations of intense erythropoiesis, radioiron uptake occurred preferentially in the large number of reticulocytes to the disadvantage of spleen or marrow erythroblasts, although they expressed comparable total numbers of TfR. Thus, it appears that when iron is scarce relative to the demand for it, circulating reticulocytes have more ready access to transferrin iron and thus reinforce iron restriction in marrow or spleen erythroblasts. However, this aggravation of functional iron deficiency only occurs in the presence of massive reticulocytosis, a finding rarely encountered in humans. Another theoretical obstacle to the use of the indirect method is when transferrin is completely saturated, allowing a significant proportion of injected radioiron to be taken up by TfRindependent mechanisms, particularly in hepatocytes but also in other nonerythroid tissues. This was not encountered in the current study. Therefore, the indirect method will remain valid in the vast majority of clinical situations.

We confirm here the excellent correlation between serum TfR levels and ETU we previously reported in rats [7] and 

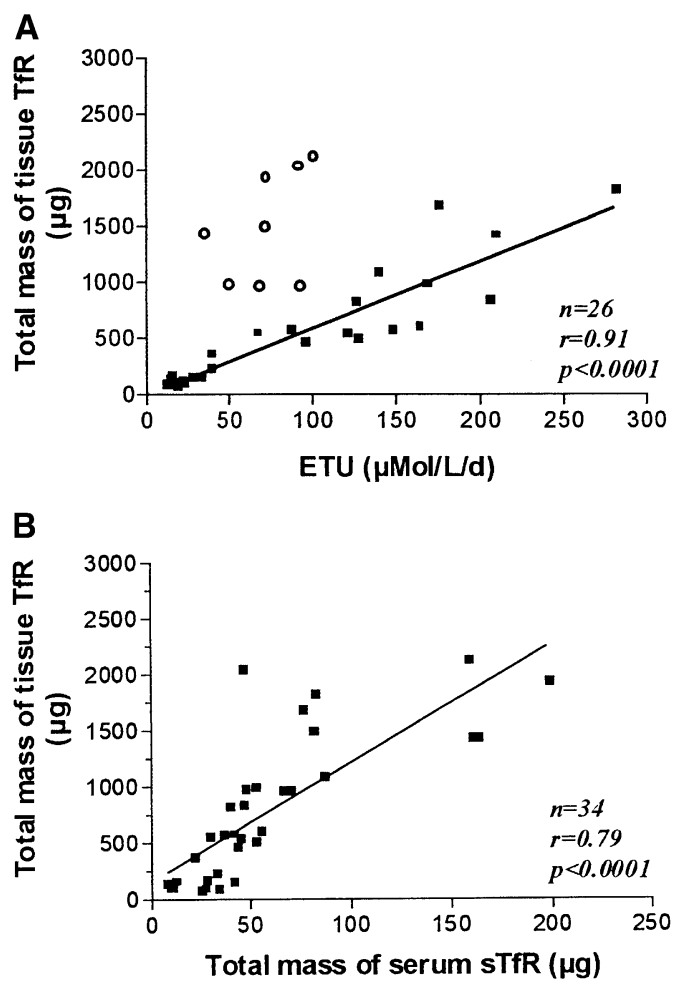

Figure 4. Correlation between the total mass of tissue TfR and the ETU (A) or the total mass of serum sTfR (B). Open circles denote the groups of animals with very low or very high serum iron that are excluded from the correlation with the ETU because the ETU was not correctly evaluated for them (see text).

in humans $[8,20,28]$. This relationship allowed development of the serum sTfR assay as a quantitative measurement of total erythropoietic activity. However, ETU will be overestimated when $\mathrm{SeFe}$ is very high because an unknown proportion of radioiron will go to nonerythroid tissues through TfR-independent mechanisms [29]. More frequently, when there are not enough transferrin-bearing molecules to saturate tissue TfR, iron uptake will be reduced and ETU will be largely underestimated. The iron supply will become inadequate when transferrin saturation is less than $20 \%$ or the rate of radioiron disappearance from the plasma is less than 25 minutes [29]. This functional iron deficiency was encountered in experiments of phlebotomy or regeneration after thiamphenicol-induced red cell aplasia.

Serum sTfR correlated very well with tissue TfR, representing $5-8 \%$ of the total mass of tissue TfR in normal animals as well as in situations of expanded or suppressed erythropoietic activity (Table 3 ). In vitro experiments with K562 or HL-60 cells evidenced parallel changes between cellular TfR and sTfR in the supernatant $[14,15]$. Soluble TfR in the supernatant was approximately $5 \%$ of the cellular TfR over a wide range of cellular iron [14]. This is remarkably similar to our results obtained in vivo. However, there were two exceptions. First, iron-restricted erythropoiesis was associated with serum sTfR/tissue TfR ratios significantly higher than in iron-replete animals. This has not been directly demonstrated during 24-hour incubation experiments in transferrin-containing medium [14]. However, the kinetics of sTfR release in vivo could be different; for instance, sTfR release could go on well beyond 24 hours in vivo if iron availability is restricted. Such a hypothesis is supported by the demonstration of a protective effect of TfR-free diferric transferrin against sTfR shedding in vitro [30]. The insufficient presence of iron-bearing transferrin relative to the number of tissue TfR could promote sTfR release in iron deficiency. The second exception is encountered in situations characterized by rapidly changing erythropoiesis. The evolution of rats treated with thiamphenicol for 4 days illustrates best the discrepancies between tissue TfR and serum sTfR in situations characterized by fast, profound changes in erythropoietic activity. Whereas the tissue TfR mass was only $20 \%$ of normal on day 4 and increased up to $3.5 \times$ normal on day 7 (Table 2), changes in serum sTfR levels could not keep up with these rapid modifications of erythropoietic activity and remained within $40 \%$ of baseline values (Fig. 2A). Ratios of serum sTfR/tissue TfR lost their meaning until the situation later stabilized (Table 3 ). Fortunately, such wild variations of erythropoiesis are seldom seen in patients, even after stem cell transplantation [31,32], and the delay between modifications in erythroblast number and serum sTfR changes is only a few days. After hypertransfusion, changes in tissue TfR occur faster than changes in serum sTfR, such that the ratio initially increases before progressively returning to baseline values.

Little is known about the rate of production and the clearance of sTfR in vivo, but the current study may provide some indirect clues. The small decrease of serum sTfR levels despite early major reductions in the number of tissue TfR in rats treated with thiamphenicol (Fig. 2A, and Tables 2 and 3), the persistence of elevated serum sTfR levels in the face of complete normalization of tissue TfR 16 days after an acute hemolytic episode (Fig. 1A, and Tables 1 and 3 ), and the continuing decrease of serum sTfR values despite stable suppression of erythropoiesis induced by polycythemia (Fig. 1C, and Tables 2 and 3) all suggest that the clearance of serum sTfR is quite slow. On the other hand, the consistent early decrease of serum sTfR values without significant change in the mass of tissue TfR in any organ suggests that an acute inflammation could accelerate the clearance of sTfR from the serum. However, it is possible that lower serum sTfR values were only the result of an expansion of plasma volume mediated by cytokines such as interleukin-6 $[33,34]$.

In conclusion, serum sTfR accurately represents a constant proportion (around 6\%) of the total mass of tissue TfR over a wide range of different rates of erythropoietic activity. However, iron-deficient erythropoiesis is associated with higher levels of serum sTfR relative to tissue TfR, and 
the pace of change in serum sTfR levels is slower than that of the tissue TfR mass.

\section{Acknowledgments}

S.R. was supported by a grant from the Fondation Frèdèricq (University of Liège, Belgium). Y.B. is Research Director of the National Fund for Scientific Research (FNRS, Belgium). This work was supported in part by grants from the FNRS.

\section{References}

1. Ponka P, Lok CN (1999) The transferrin receptor: role in health and disease. Int J Biochem Cell Biol 31:1111

2. Lok CN, Loh TT (1998) Regulation of transferrin function and expression: review and update. Biol Signals Recept 7:157

3. Huebers HA, Finch CA (1987) The physiology of transferrin and transferrin receptors. Physiol Rev 67:520

4. Loken MR, Shah VO, Dattilio KL, Civin CI (1987) Flow cytometric analysis of human bone marrow: I. Normal erythroid development. Blood 69:255

5. Iacopetta BJ, Morgan EH, Yeoh GCT (1982) Transferrin receptors and iron uptake during erythroid cell development. Biochim Biophys Acta 687:204

6. van Bockxmeer FM, Morgan EH (1979) Transferrin receptors during rabbit reticulocyte maturation. Biochim Biophys Acta 584:76

7. Beguin Y, Huebers HA, Josephson B, Finch CA (1988) Transferrin receptors in rat plasma. Proc Natl Acad Sci U S A 85:637

8. Huebers HA, Beguin Y, Pootrakul P, Einspahr D, Finch CA (1990) Intact transferrin receptors in human plasma and their relation to erythropoiesis. Blood 75:102

9. Shih YJ, Baynes RD, Hudson BG, Flowers CH, Skikne BS, Cook JD (1990) Serum transferrin receptor is a truncated form of tissue receptor. J Biol Chem 265:19077

10. Skikne BS, Flowers CH, Cook JD (1990) Serum transferrin receptor: a quantitative measure of tissue iron deficiency. Blood 75:1870

11. Suominen P, Punnonen K, Rajamaki A, Irjala K (1998) Serum transferrin receptor and transferrin receptor-ferritin index identify healthy subjects with subclinical iron deficits. Blood 92:2934

12. Cazzola M, Huebers HA, Sayers MH, MacPhail AP, Eng M, Finch CA (1985) Transferrin saturation, plasma iron turnover, and transferrin uptake in normal humans. Blood 66:935

13. Cazzola M, Pootrakul P, Huebers HA, Eng M, Eschbach J, Finch CA (1987) Erythroid marrow function in anemic patients. Blood 69:296

14. Baynes RD, Shih YJ, Cook JD (1991) Production of soluble transferrin receptor by K562 erythroleukaemia cells. Br J Haematol 78:450

15. Chitambar CR, Zivkovic Gilgenbach Z (1990) Influence of cellular iron status on the release of soluble transferrin receptor from human promyelocytic leukemic HL60 cells. J Lab Clin Med 116:345

16. Cook JD, Marsaglia G, Eschbach JW, Funk DD, Finch CA (1970) Ferrokinetics: a biologic model for plasma iron exchange in man. J Clin Invest 49:197
17. Beguin Y, Bergamaschi G, Huebers HA, Finch CA (1988) The behavior of asialotransferrin-iron in the rat. Am J Hematol 29:204

18. Hershko C, Cook JD, Finch CA (1972) Storage iron kinetics. II. The uptake of hemoglobin iron by hepatic parenchymal cells. J Lab Clin Med 80:624

19. Lee LG, Chen CH, Chiu LA (1986) Thiazole orange: a new dye for reticulocyte analysis. Cytometry 7:508

20. Beguin Y, Clemons G, Pootrakul P, Fillet G (1993) Quantitative assessment of erythropoiesis and functional classification of anemia based on measurements of serum transferrin receptor and erythropoietin. Blood 81:1067

21. Iron Panel of the International Committee for Standardization in Haematology (1990) Revised recommendations for the measurements of the serum iron in human blood. Br J Haematol 75:615

22. de Haan G, Dontje B, Nijhof W, Loeffler M (1995) Effects of continuous stem cell factor administration on normal and erythropoietin-stimulated murine hemopoiesis: experimental results and model analysis. Stem Cells 13:65

23. Goris H, Bungart B, Loeffler M, Schmitz S, Nijhof W (1990) Migration of stem cells and progenitors between marrow and spleen following thiamphenicol treatment of mice. Exp Hematol 18:400

24. Brandan N, Aguirre M, Carmuega R, Alvarez M, Juaristi J (1997) Proliferative and maturative behaviour patterns on murine bone marrow and spleen erythropoiesis along hypoxia. Acta Physiol Pharmacol Ther Latinoam 47:125

25. Boggs DR, Geist A, Chervenick PA (1969) Contribution of the mouse spleen to post-hemorrhagic erythropoiesis. Life Sci 8:587

26. Bozzini CE, Barrio Rendo ME, Devoto FC, Epper CE (1970) Studies on medullary and extramedullary erythropoiesis in the adult mouse. Am J Physiol 219:724

27. Haile DJ (1999) Regulation of genes of iron metabolism by the ironresponse proteins. Am J Med Sci 318:230

28. Beguin Y (1992) The soluble transferrin receptor: biological aspects and clinical usefulness as quantitative measure of erythropoiesis. Haematologica 77:1

29. Beguin Y, Stray SM, Cazzola M, Huebers HA, Finch CA (1988) Ferrokinetic measurement of erythropoiesis. Acta Haematol 79:121

30. Baynes RD (1995) Transferrin reduces the production of soluble transferrin receptor. Proc Soc Exp Biol Med 209:286

31. Beguin Y, Baron F, Fillet G (1998) Influence of marrow erythropoietic activity on serum erythropoietin levels after autologous hematopoietic stem cell transplantation. Haematologica 83:1076

32. Beguin Y, Oris R, Fillet G (1993) Dynamics of erythropoietic recovery after bone marrow transplantation: role of marrow proliferative capacity and erythropoietin production in autologous versus allogeneic transplants. Bone Marrow Transplant 11:285

33. Atkins MB, Kappler K, Mier JW, Isaacs RE, Berkman EM (1995) Interleukin-6-associated anemia: determination of the underlying mechanism. Blood 86:1288

34. Nieken J, Mulder NH, Buter J, et al. (1995) Recombinant human interleukin-6 induces a rapid and reversible anemia in cancer patients. Blood 86:900 